\title{
Linear angular dispersion compensation of cleaned self-diffraction light with a single prism
}

\author{
Xiong Shen ${ }^{1,2}$, Peng Wang ${ }^{1,2}$, Jun $\mathrm{Liu}^{1,3}$, and Ruxin $\mathrm{Li}^{1,3}$ \\ ${ }^{1}$ State Key Laboratory of High Field Laser Physics, Shanghai Institute of Optics and Fine Mechanics, Chinese Academy of Sciences, \\ Shanghai 201800, China \\ ${ }^{2}$ University of Chinese Academy of Sciences, Beijing 100049, China \\ ${ }^{3}$ IFSA Collaborative Innovation Center, Shanghai Jiao Tong University, Shanghai 200240, China \\ (Received 2 November 2017; revised 9 February 2018; accepted 7 March 2018)
}

\begin{abstract}
The linear angular dispersion of a self-diffraction (SD) pulse, from a femtosecond laser pulse cleaning device, is compensated for by the use of a single prism. More than $500 \mu \mathrm{J}$ first-order SD pulse has a contrast of $10^{12}$, which is about five orders of magnitude improvement from the input fundamental pulse. The wings of the distribution away from the main pulse in \pm 1 ps are cleaned with a contrast improvement of about $10^{7}$, which verifies the pulse cleaning ability of the SD process.
\end{abstract}

Keywords: angular dispersion; femtosecond; self-diffraction; temporal contrast

\section{Introduction}

Peta watt (PW) level laser systems, with focused laser peak intensity of about $10^{22} \mathrm{~W} / \mathrm{cm}^{2}$ or even higher, have been reported repeatedly ${ }^{[1-6]}$. These extremely high intensity conditions have aroused new interest in exploring lasermatter interactions, such as particle acceleration, bright $\mathrm{X}$-ray source generation, and inertial confinement fusion ${ }^{[7,8]}$. Temporal contrast is one of the crucial parameters of PW laser pulses. Prepulses with laser peak intensity higher than $10^{10} \mathrm{~W} / \mathrm{cm}^{2[9,10]}$ in advance of the main pulse should be avoided as they can pre-ionize the target and generate a low density preplasma before the main pulse reaches the target.

To build high temporal contrast laser systems, optical parametric chirped-pulse amplification (OPCPA) $)^{[11,12]}$ and double chirped-pulse amplification (double CPA) ${ }^{[3,13,14]}$ combined with pulse cleaning techniques have been proposed in the past decades. These pulse cleaning techniques include saturable absorbers ${ }^{[15]}$, optical parametric amplification $^{[3,16]}$, polarization rotation ${ }^{[13,17]}$, and crosspolarized wave (XPW) generation ${ }^{[18-20]}$. Pulse cleaning by using a self-diffraction (SD) process in a bulk Kerr medium was proposed recently ${ }^{[21,22]}$.

In theory, the temporal contrast of the first-order SD signal is the cube of the temporal contrast of the incident pulse as

Correspondence to: J. Liu, No. 390 Qinghe Road, Jiading, Shanghai 201800, China. Email: jliu@siom.ac.cn
$C_{S D \pm 1} \approx C_{i n}^{3}$, because the generated cleaned SD signals are spatially separated from the incident beams without use of any polarization discrimination devices. What is more, this technique can also achieve a high energy SD signal output using a cylinder mirror focusing on the incident beams, even with low energy-conversion efficiency ${ }^{[21]}$. These two advantages make the SD process a good choice for providing seed pulses for PW laser systems. However, the angular dispersion of the SD signals may affect the pulse compression of the amplified pulse in the compressor.

In this study, we report the highest pulse energy and highest temporal contrast enhancement pulses generation based on the SD effect with two cylindrical convex lenses so far. More than $500 \mu \mathrm{J}$ first-order SD signal with a temporal contrast of $10^{12}$ is generated with about five orders of magnitude improvement. The wings around the main pulse in $\pm 1 \mathrm{ps}$ are cleaned with a contrast improvement of about $10^{7}$, which verifies the pulse cleaning ability of SD process. The cause of angular dispersion generation of the SD signals is also explored, and the linear angular dispersion is compensated with a single prism.

\section{Principle and experimental setup}

The SD process is a degenerated cascaded four-wave mixing (DCFWM) process ${ }^{[21-23]}$. It acts as a temporal gating to improve the temporal contrast of a laser pulse. As an 


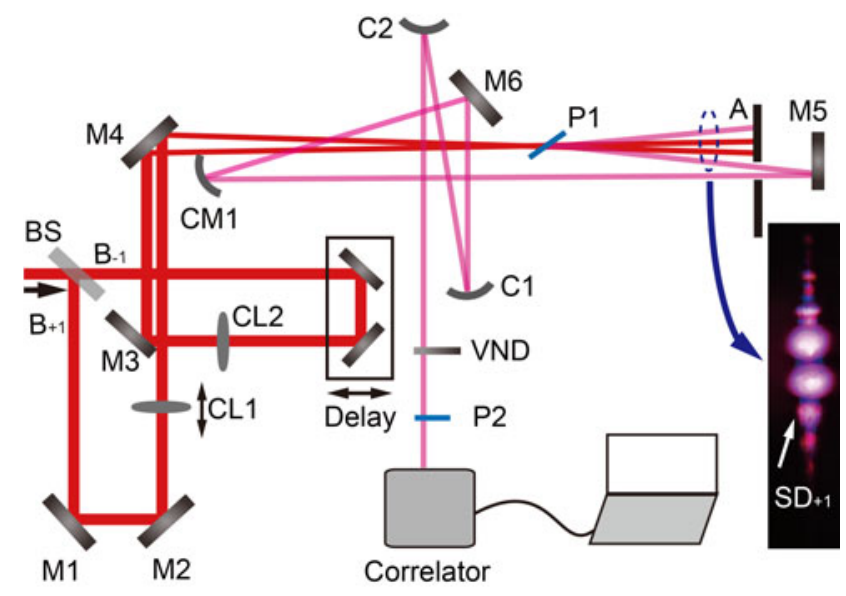

Figure 1. Experimental setup for self-diffraction signal generation and temporal contrast measurement. BS: beam splitter; M1-M6: reflective mirrors; CL1, CL2: plane-convex cylindrical lenses, $f=500 \mathrm{~mm}$; P1: $0.15 \mathrm{~mm}$ thick fused silica plate; A: aperture; CM1: cylindrical reflective mirror, $f=500 \mathrm{~mm}$; $\mathrm{C} 1$ : spherical concave reflective mirror, $f=$ $-500 \mathrm{~mm}$; C2: spherical convex reflective mirror, $f=200 \mathrm{~mm}$; VND: $2 \mathrm{~mm}$ thick variable neutral-density filter; P2: $1 \mathrm{~mm}$ thick fused silica plate; correlator: third-order cross-correlator (Amplitude Technologies Inc., Sequia 800).

instantaneous third-order nonlinear optical effect, the SD process happened in hundreds of femtosecond time scale which requires a relatively high laser intensity at the same time. Weak satellite pulses and amplified spontaneous emission (ASE) noise can be filtered out by this process ${ }^{[21]}$. In the time domain, the intensity of the first-order SD signal has the relationship to the intensities of the two input beams as $I_{S D+1} \propto I_{+1}^{2}(t) I_{-1}(t-\tau)$, where $\tau$ is the time delay between the two input pulses ${ }^{[24]}$. According to the equation, the temporal contrast of the $S D_{+1}$ signal is the cube of that of the incident pulse $C_{S D+1} \approx C_{i n}^{3}$. Furthermore, the generated signals and incident beams are separated spatially, which means no polarizer with a limited extinction ratio is needed, which is a main limitation for the XPW process. These properties indicate a potential high temporal contrast enhancement by using the SD process.

Figure 1 shows the experimental setup. Pulses from a laser system are separated into two beams $B_{+}$and $B_{-}$by a $50 / 50$ beam splitter. Both $B_{+}$and $B_{-}$are separately focused into a Kerr medium P1 by two plane-convex cylindrical lenses CL1 and CL2. There is a delay line in the path of $B_{-}$. P1 is a $0.15 \mathrm{~mm}$ thick fused silica plate which is located about $60 \mathrm{~mm}$ behind the focus of the two lenses with a near Brewster angle to the two incident beams. The external crossing angle $\theta$ between the two incident beams is about $1.6^{\circ}$, where the phase-matching condition can be easily satisfied ${ }^{[21]}$. SD signals are formed beside the two incident beams in the condition that the two beams are temporally and spatially overlapped. An aperture A filters out the first-order SD signal $S D_{+1}$, then $S D_{+1}$ is collimated by a cylindrical reflective mirror CM1. The collimated $S D_{+1}$

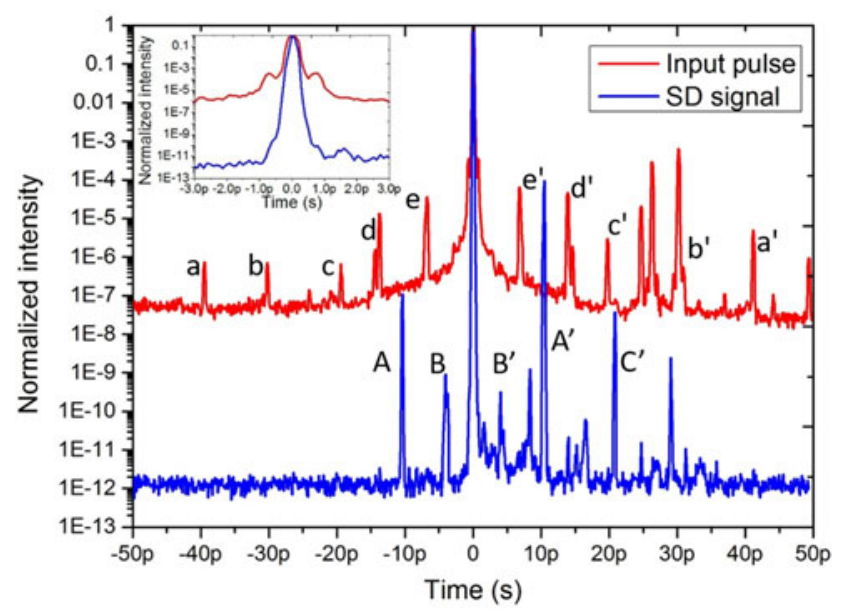

Figure 2. The temporal contrast of the input pulse and the generated firstorder SD signal. The inset is an enlarged part of the curves from $-3 \mathrm{ps}$ to 3 ps.

beam is reduced in beam size to about $3 \mathrm{~mm}$ diameter by a spherical concave reflective mirror and a spherical convex reflective mirror, and is then taken to a commercial thirdorder cross-correlator (Amplitude Technologies Inc., Sequia 800) for temporal contrast measurement. A variable neutraldensity (VND) filter is used to adjust the input energy into the correlator. P2 is inserted to create replicas of the main pulse of $S D_{+1}$.

\section{Results and discussion}

\subsection{High energy and high temporal contrast SD pulse generation}

To generate SD signals with high energy and temporal contrast, experiments were performed with a commercial Ti:sapphire CPA laser system (Legend Elite Cryo PA, Coherent Inc.). The laser system produces $1 \mathrm{kHz} / 50 \mathrm{fs} / 800 \mathrm{~nm} /$ $10 \mathrm{~mJ}$ pulses with a diameter of about $15 \mathrm{~mm}$. The pulse energies of the two incident beams before the Kerr medium P1 are both about $4.9 \mathrm{~mJ}$. The beam size on the Kerr medium is about $15 \mathrm{~mm} \times 1.2 \mathrm{~mm}$. About five diffracted orders of SD signals are generated beside each side of the two incident beams. The first-order SD signals $S D_{+1}$ and $S D_{-1}$ are about $780 \mu \mathrm{J}$ and $630 \mu \mathrm{J}$, respectively. The energyconversion efficiency from the two incident beams to $S D_{+1}$ is about $7.8 \%$.

To characterize the temporal contrast of $S D_{+1}$, the diameter of signal $S D_{+1}$ beam is reduced to about $3 \mathrm{~mm}$ by C1 (spherical concave reflective mirror, $f=-500$ ) and $\mathrm{C} 2$ (spherical convex reflective mirror, $f=200 \mathrm{~mm}$ ) first. Then, a $2 \mathrm{~mm}$ thick VND filter is used to adjust the input energy to the correlator to about $200 \mathrm{~mW}$. A $1 \mathrm{~mm}$ thick fused silica plate is inserted in the path of $S D_{+1}$ to introduce reference post-pulses. 


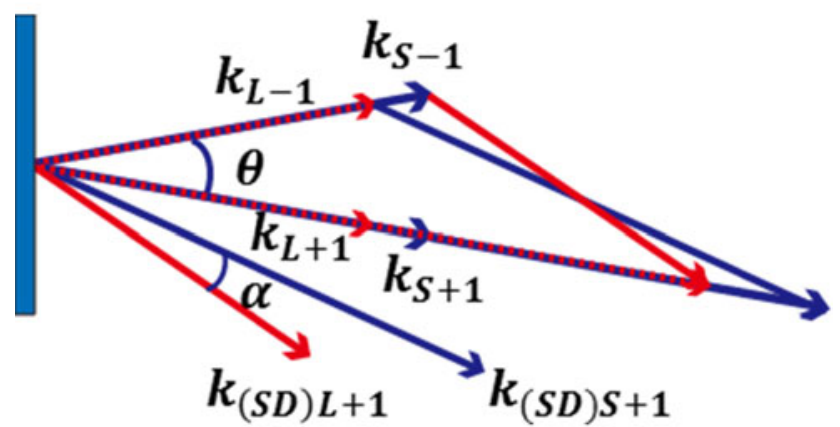

Figure 3. Scheme of angular dispersion generation.

The measured temporal contrast curves of the input pulse and $S D_{+1}$ are shown in Figure 2. For the input pulse, before the main pulse, there are five replicas a/b/c/d/e with contrast varying from $10^{6}$ to $10^{4}$, and they are time symmetrical with replicas a'/b'/c'/d'/e' after the main pulse, respectively. These replicas may come from reflection between front and rear surfaces of optical elements in the laser path. An amplified ASE-noise with contrast change from $10^{7}$ to $10^{4}$ also exists around the main pulse. The $1 \mathrm{~mm}$ thick fused silica plate $\mathrm{P} 2$ introduces replicas A' and C' after the main pulse of $S D_{+1}$ with normalized intensity about $10^{-4}$ and $10^{-8}$, respectively, which are about one order lower than the values introduced by the front and rear sides reflection of a fused silica plate. It may be caused by the tilt of P2, that the replicas and the main pulse are not collinear entirely. Replicas A and B are time symmetrical with replicas A' and B'. From the inset in Figure 2, we can see that the SD process lasting just hundreds of femtosecond gated out all the replicas before the main pulse of the input pulse. Wings around the main pulse in \pm 1 ps are cleaned with a contrast improvement of about $10^{7}$, which verifies the pulse cleaning ability of SD process.

\subsection{Analyzation of angular dispersion generation}

The generation of the angular dispersion of $S D_{+1}$ is caused by the different phase-matching condition of wavelength components of the two incident beams shown in Figure 3.

For $S D_{+1}$, its phase-matching condition is $\boldsymbol{k}_{S D+1}=$ $2 \boldsymbol{k}_{+1}-\boldsymbol{k}_{-1}$. In the $\boldsymbol{k}_{-1}$ direction, $\boldsymbol{k}_{L-1}$ denotes the wave vector of the longest wavelength component and $\boldsymbol{k}_{S-1}$ the wave vector of the shortest wavelength component. Similarly, in the $\boldsymbol{k}_{+1}$ direction, $\boldsymbol{k}_{L+1}$ denotes the wave vector of the longest wavelength component and $\boldsymbol{k}_{S+1}$ the wave vector of the shortest wavelength component. Then the generated longest wavelength $\lambda_{N L}$ and shortest wavelength $\lambda_{N S}$ in $S D_{+1}$ can be expressed as $\lambda_{N L}=$ $1 / \operatorname{sqrt}\left(\lambda_{S}^{-2}+4 \lambda_{L}^{-2}-4 \lambda_{S}^{-1} \cdot \lambda_{L}^{-1} \cdot \cos \theta\right)$ and $\lambda_{N S}=$ $1 / \operatorname{sqrt}\left(\lambda_{L}^{-2}+4 \lambda_{S}^{-2}-4 \lambda_{S}^{-1} \cdot \lambda_{L}^{-1} \cdot \cos \theta\right)$, respectively. $\lambda_{L}$ is the original longest and $\lambda_{S}$ the shortest wavelength of the incident pulses. The phase-matching conditions for the longest and shortest wavelength SD signals are $\boldsymbol{k}_{(S D) L+1}=$ $2 \boldsymbol{k}_{L+1}-\boldsymbol{k}_{S-1}$ and $\boldsymbol{k}_{(S D) S+1}=2 \boldsymbol{k}_{S+1}-\boldsymbol{k}_{L-1}$, respectively. Then the dispersion angle $\alpha$ of $S D_{+1}$ is the angle between $\boldsymbol{k}_{(S D) L+1}$ and $\boldsymbol{k}_{(S D) S+1}$. It is related to the cross angle of the two incident beams, the shortest and the longest wavelength components of the two beams, and can be expressed as $\alpha=a-b, a=\arcsin \left\{2 k_{S+1} \sin \theta / \operatorname{sqrt}\left[k_{L-1}^{2}+\left(2 k_{S+1}\right)^{2}-\right.\right.$ $\left.\left.4 k_{S+1} k_{L-1} \cos \theta\right]\right\}, \quad b=\arcsin \left\{2 k_{L+1} \sin \theta / \operatorname{sqrt}\left[k_{S-1}^{2}+\right.\right.$ $\left.\left.\left(2 k_{L+1}\right)^{2}-4 k_{S-1} k_{L+1} \cos \theta\right]\right\}$.

There is a little difference between the center wavelength of $S D_{+1}$ and that of the incident pulses, and can be calculated according to the law of cosines: $\lambda_{N}=\lambda_{O} / \operatorname{sqrt}(5-$ $4 \cos \theta$ ), where $\lambda_{N}$ is the new generated center wavelength of $S D_{+1}$ and $\lambda_{O}$ the original center wavelength of the incident pulses. For $\lambda_{O}=803 \mathrm{~nm}, \theta=1.6^{\circ}$. The new generated center wavelength of $S D_{+1} \lambda_{N} \approx 802.37 \mathrm{~nm}$. It can be concluded that for a small crossing angle of the two incident beams, the generated first-order SD signals almost keep the same center wavelength of the incident pulses, and the SD process can be looked on as a frequency-conserving process.

\subsection{Angular dispersion compensation}

For an incident beam with a spectrum as shown in Figure 4(a), its full width at half maximum (FWHM) spectrum width $w$ is about $28 \mathrm{~nm}$ with center wavelength at $803 \mathrm{~nm}$. The generated dispersion angle $\alpha \approx 3.90 \mathrm{mrad}$ according to the equation of $\alpha$. Then the angular dispersion can be roughly calculated as $A_{c}=\alpha / w \approx 0.14 \mathrm{mrad} / \mathrm{nm}$. The divergence angle caused by the lenses CL1 and CL2 with $500 \mathrm{~mm}$ focal length is about $\beta \approx \arcsin (6 / 500) \approx 12 \mathrm{mrad}$. After about $2000 \mathrm{~mm}$, the beam width of the $S D_{+1}$ is about $l=2000 \mathrm{~mm} \times \sin (\alpha+\beta) \approx 32 \mathrm{~mm}$.

In the experiment, after propagation of about $2000 \mathrm{~mm}$ in air, the width of beam $S D_{+1}$ is about $30 \mathrm{~mm}$. We measured the spectra of $S D_{+1}$ at 30 different positions P0 to P29 in the horizontal direction, with every two positions separated by about $1 \mathrm{~mm}$. Figure 4(b) shows the spectra measured at position $\mathrm{P} 0 / \mathrm{P} 4 / \mathrm{P} 9 / \mathrm{P} 14 / \mathrm{P} 19 / \mathrm{P} 24 / \mathrm{P} 29$ points. The curve of center wavelength measured at each position is presented in the insert figure in Figure 4(b). The center wavelength is shifted by about $20 \mathrm{~nm}$. The angular dispersion measured can be roughly denoted as $A_{m}=[\arcsin (30 / 2000)-$ $\beta] \mathrm{rad} / 20 \mathrm{~nm} \approx 0.15 \mathrm{mrad} / \mathrm{nm} . A_{m} \approx A_{c}$, the measured result of the angular dispersion of generated SD signal matches very well with the theoretically calculated result.

The scheme of angular dispersion compensation is shown in Figure 5. A fused silica prism with apex angle $\alpha=\pi / 3$ rad is used to compensate the angular dispersion in our experiment. In beam $S D_{+1}$, longer wavelength is located far away from the two incident beams and shorter wavelength located closer to the two incident beams. A cylindrical reflective mirror with focal length of $200 \mathrm{~mm}$ located $400 \mathrm{~mm}$ 

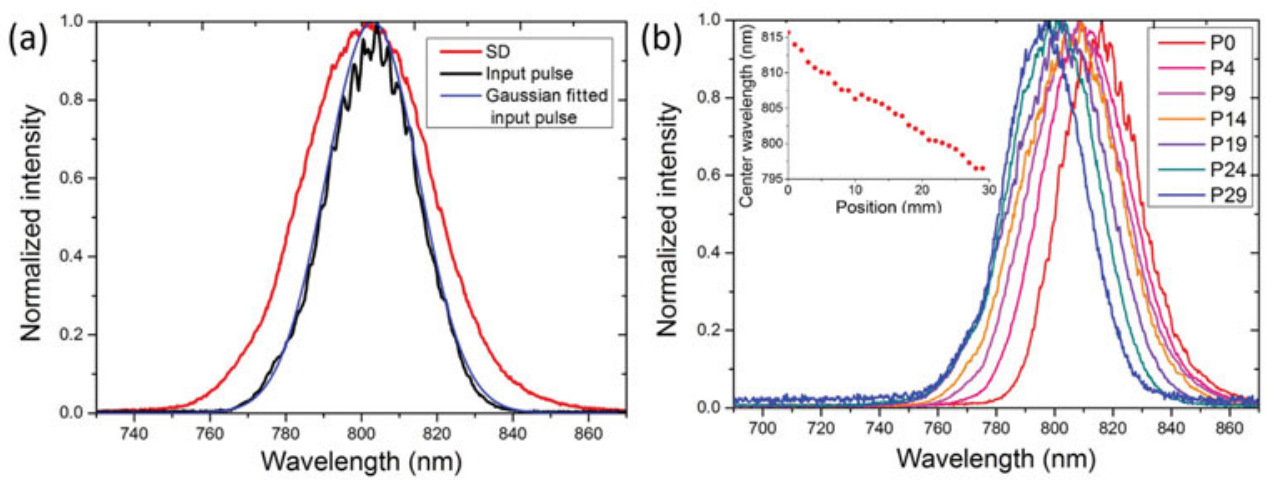

Figure 4. (a) The spectra of the incident pulse and the first-order SD signal $S D_{+1}$; (b) the $S D_{+1}$ spectra at 30 different positions, the inset shows the center wavelengths measured at each position.

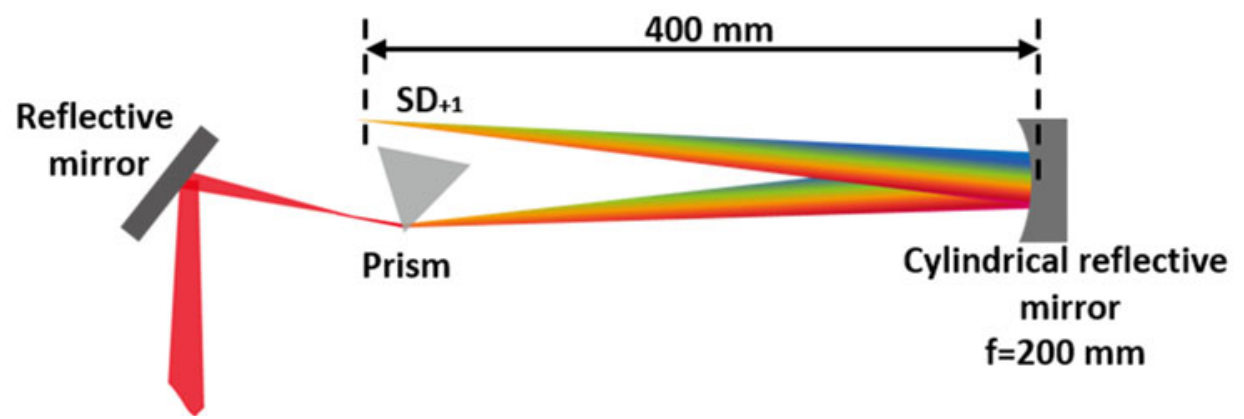

Figure 5. Scheme of angular dispersion compensation.

behind the Kerr medium P1 is used to symmetrically focus $S D_{+1}$.

For a prism with refractive index $n$ and apex angle $\alpha$, we can obtain the angular dispersion of the prism ${ }^{[25]}$ as $\mathrm{d} \emptyset_{2} / \mathrm{d} n=\left(\cos \emptyset_{2}\right)^{-1}\left[\sin \left(\emptyset_{2}^{\prime}\right)+\cos \left(\emptyset_{2}^{\prime}\right) \tan \left(\emptyset_{1}^{\prime}\right)\right]$. According to the measurement, the angular dispersion of the generated $S D_{+1}$ is about $0.15 \mathrm{mrad} / \mathrm{nm}$ with center wavelength shifting from $795 \mathrm{~nm}$ to $815 \mathrm{~nm}$. We just consider the two edge center wavelengths $\lambda_{N S}=795 \mathrm{~nm}$ and $\lambda_{N L}=815 \mathrm{~nm}$ here. We can calculate their refractive index according to Cauchy's dispersion formula in the prism as $n_{N S}=1.4534$ and $n_{N L}=1.4531$, respectively.

The relation between $\emptyset_{1}$ and $\emptyset_{2}$ can be calculated with Snell's law $\sin \emptyset_{1}=n \sin \emptyset_{1}^{\prime}, \sin \emptyset_{2}=n \sin \emptyset_{2}^{\prime}$ and $\emptyset_{1}^{\prime}+\emptyset_{2}^{\prime}=$ $\alpha=\pi / 3 \mathrm{rad}$. If the cross angle between the two output beams is about $\beta=0.15 \mathrm{mrad} / \mathrm{nm} \times 20 \mathrm{~nm}=3 \mathrm{mrad}$, the angle $\emptyset_{1}=0.432 \mathrm{rad}$ is the right output angle of the $S D_{+1}$ beam. The input angle of the $S D_{+1}$ beam can be calculated as about $\emptyset_{2}=1.481 \mathrm{rad}$.

The angular dispersion compensated $S D_{+1}$ propagates about $1500 \mathrm{~mm}$, and is expanded to about $20 \mathrm{~mm}$ in the horizontal direction. The spectra of $S D_{+1}$ at five different positions with $5 \mathrm{~mm}$ apart are shown in Figure 6(c).

As can be seen in Figure 6(c), the spectra of compensated $S D_{+1}$ at five different positions show a good coincidence. While, residual angular dispersion still exists, and it is clear that the compensation for the shorter wavelengths appears better than that for the longer wavelengths, we think this is the residual high-order angular dispersion as the center wavelengths vary nonlinearly relative to the five positions of equal interval.

\section{Conclusion}

In conclusion, temporal contrast enhancement by the SD process in a bulk Kerr medium possesses a few advantages compared to many other pulse cleaning techniques. The SD signals are spatially separated from the incident beams without the use of any polarization discrimination devices. It can also achieve a high energy SD signal output with a cylindrical mirror focusing on the incident beams even with low energy-conversion efficiency. The temporal contrast of the $S D_{+1}$ signal is the cube of the temporal contrast of the incident pulse, $C_{S D+1} \approx C_{i n}^{3}$ in theory, which indicates a great potential of temporal contrast enhancement by the SD process.

In this study, a temporal contrast enhancement equipment based on SD effect with two cylindrical convex lenses is built. As high as $780 \mu \mathrm{J}$ first-order SD signal at $800 \mathrm{~nm}$ with contrast of $10^{12}$ is generated with about five orders of magnitude improvement. Wings around the main pulse 
(a)

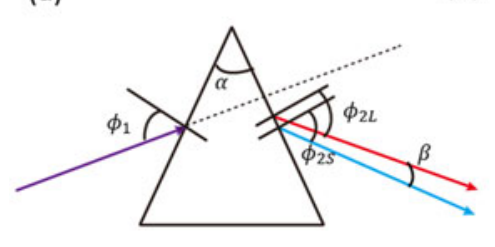

(b)

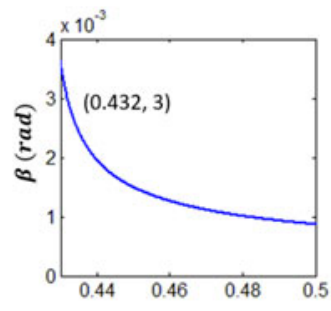

$\alpha=\pi / 3 \mathrm{rad}$

(c)

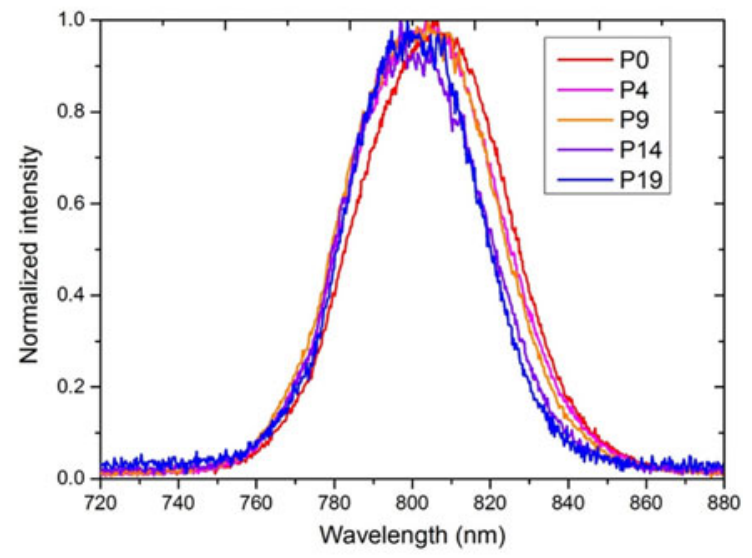

Figure 6. (a) Scheme of prism dispersion; (b) the relationship between the incident angle $\emptyset_{1}$ and the dispersion angle of pulses with $\lambda_{N S}=795 \mathrm{~nm}$ and $\lambda_{N L}=815 \mathrm{~nm}$; (c) spectra of compensated SD signal.

in \pm 1 ps are cleaned with a contrast improvement of about $10^{7}$, which verifies the pulse cleaning ability of SD process. The cause of angular dispersion generation of the SD signals is also explored, and the angular dispersion is compensated with a single prism. It is expected to extend the SD process as an effective pulse cleaning method for high power laser at $1053 \mathrm{~nm}$ with a narrow spectral bandwidth and hundreds of femtosecond pulse duration.

\section{Acknowledgements}

The authors would like to thank Wei Chu and Ziting Li for the support of laser sources, and Yi Xu for his help on temporal contrast measurement. This work is supported by the National Natural Science Foundation of China (NSFC) (Nos. 11274327, 61521093, and 61527821), the Instrument Developing Project of the Chinese Academy of Sciences (No. YZ201538), and the Strategic Priority Research Program of the Chinese Academy of Sciences (No. XDB16).

\section{References}

1. M. Aoyama, K. Yamakawa, Y. Akahane, J. Ma, N. Inoue, H. Ueda, and H. Kiriyama, Opt. Lett. 28, 1594 (2003).
2. V. V. Lozhkarev, G. I. Freidman, V. N. Ginzburg, E. V. Katin, E. A. Khazanov, A. V. Kirsanov, G. A. Luchinin, A. N. Mal'shakov, M. A. Martyanov, O. V. Palashov, A. K. Poteomkin, A. M. Sergeev, A. A. Shaykin, and I. V. Yakovlev, Laser Phys. Lett. 4, 421 (2007).

3. Z. Wang, C. Liu, Z. Shen, Q. Zhang, H. Teng, and Z. Wei, Opt. Lett. 36, 3194 (2011).

4. T. J. Yu, S. K. Lee, J. H. Sung, J. W. Yoon, T. M. Jeong, and J. Lee, Opt. Express 20, 10807 (2012).

5. Y. Chu, Z. Gan, X. Liang, L. Yu, X. Lu, C. Wang, X. Wang, L. Xu, H. Lu, D. Yin, Y. Leng, R. Li, and Z. Xu, Opt. Lett. 40, 5011 (2015).

6. L. Yu, X. Liang, L. Xu, W. Li, C. Peng, Z. Hu, C. Wang, X. Lu, Y. Chu, Z. Gan, X. Liu, Y. Liu, X. Wang, H. Lu, D. Yin, Y. Leng, R. Li, and Z. Xu, Opt. Lett. 40, 3412 (2015).

7. H. Kiriyama, M. Mori, A. S. Pirozhkov, K. Ogura, A. Sagisaka, A. Kon, T. Z. Esirkepov, Y. Hayashi, H. Kotaki, M. Kanasaki, H. Sakaki, Y. Fukuda, J. Koga, M. Nishiuchi, M. Kando, S. V. Bulanov, K. Kondo, P. R. Bolton, O. Slezak, D. Vojna, M. Sawicka-Chyla, V. Jambunathan, A. Lucianetti, and T. Mocek, IEEE J. Sel. Top. Quantum Electron. 21, 18 (2015).

8. Y. I. Salamin, S. X. Hu, K. Z. Hatsagortsyan, and C. H. Keitel, Phys. Rep. 427, 41 (2006).

9. P. B. Corkum, F. Brunel, N. K. Sherman, and T. Srinivasanrao, Phys. Rev. Lett. 61, 2886 (1988).

10. B. C. Stuart, M. D. Feit, S. Herman, A. M. Rubenchik, B. W. Shore, and M. D. Perry, Phys. Rev. B 53, 1749 (1996).

11. H. Kiriyama, M. Mori, Y. Nakai, Y. Yamamoto, M. Tanoue, A. Akutsu, T. Shimomura, S. Kondo, S. Kanazawa, H. Daido, T. Kimura, and N. Miyanaga, Opt. Lett. 32, 2315 (2007).

12. H. Kiriyama, M. Mori, Y. Nakai, T. Shimomura, M. Tanoue, A. Akutsu, S. Kondo, S. Kanazawa, H. Okada, T. Motomura, H. Daido, T. Kimura, and T. Tajima, Opt. Lett. 33, 645 (2008).

13. M. P. Kalashnikov, E. Risse, H. Schonnagel, and W. Sandner, Opt. Lett. 30, 923 (2005).

14. N. Xie, K. N. Zhou, W. Q. Huang, X. D. Wang, L. Sun, Y. Guo, and Q. Li, Opt. Eng. 51, 5 (2012).

15. H. Kiriyama, M. Mori, Y. Nakai, T. Shimomura, H. Sasao, M. Tanoue, S. Kanazawa, D. Wakai, F. Sasao, H. Okada, I. Daito, M. Suzuki, S. Kondo, K. Kondo, A. Sugiyama, P. R. Bolton, A. Yokoyama, H. Daido, S. Kawanishi, T. Kimura, and T. Tajima, Opt. Lett. 35, 1497 (2010).

16. Y. S. Huang, C. M. Zhang, Y. Xu, D. X. Li, Y. X. Leng, R. X. Li, and Z. Z. Xu, Opt. Lett. 36, 781 (2011).

17. D. Homoelle, A. L. Gaeta, V. Yanovsky, and G. Mourou, Opt. Lett. 27, 1646 (2002).

18. V. Yanovsky, V. Chvykov, G. Kalinchenko, P. Rousseau, T. Planchon, T. Matsuoka, A. Maksimchuk, J. Nees, G. Cheriaux, G. Mourou, and K. Krushelnick, Opt. Express 16, 2109 (2008).

19. V. Chvykov, P. Rousseau, S. Reed, G. Kalinchenko, and V. Yanovsky, Opt. Lett. 31, 1456 (2006).

20. A. Jullien, O. Albert, F. Burgy, G. Hamoniaux, L. P. Rousseau, J. P. Chambaret, F. Auge-Rochereau, G. Cheriaux, J. Etchepare, N. Minkovski, and S. M. Saltiel, Opt. Lett. 30, 920 (2005).

21. F. J. Li, X. Shen, P. Wang, Y. Y. Li, J. Liu, Z. S. Wang, and R. X. Li, Laser Phys. Lett. 13, 055303 (2016).

22. J. Liu, K. Okamura, Y. Kida, and T. Kobayashi, Opt. Express 18, 22245 (2010).

23. J. Liu and T. Kobayashi, Sensors 10, 4296 (2010).

24. V. L. Vinetskii, N. V. Kukhtarev, S. G. Odulov, and M. S. Soskin, Usp. Fiz. Nauk 129, 113 (1979).

25. R. L. Fork, O. E. Martinez, and J. P. Gordon, Opt. Lett. 9, 150 (1984). 